\title{
Qué contenidos y qué habilidades cognitivo-lingüísticas emplea el profesorado de primaria y secundaria en la enseñanza de la astronomía
}

\author{
What content and which cognitive-linguistic skills are used \\ by primary and secondary school teachers when teaching
} astronomy

Concepción González Rodríguez, Susana García Barros, Cristina Martínez Losada Facultade de Ciencias da Educación. Universidade da Coruña. España. cgonzalezr@udc.es, susg@udc.es y cmarl@udc.es

RESUMEN • Se analizan 373 actividades de enseñanza y 103 cuestiones de evaluación dirigidas al estudio de la astronomía en la educación obligatoria, aportadas por 14 profesores ( 7 de primaria y 7 de secundaria). Con este análisis se pretende conocer qué aspectos conceptuales y qué habilidades cognitivo-lingüísticas se incluyen en ellas. Los resultados muestran que el profesorado tiende a priorizar el estudio de los aspectos más complejos -la Tierra en el espacio- y, en lo referente a las habilidades cognitivo-lingüísticas, a solicitar la descripción de modelos teóricos. Además, se aprecia cierto desequilibrio en el uso de dichas habilidades en relación con el estudio de distintos aspectos astronómicos. Finalmente, se presentan recomendaciones didácticas derivadas de los resultados.

PALABRAS CLAVE: astronomía; habilidades cognitivo-lingüísticas; actividades; profesorado de primaria y secundaria.

ABSTRACT $\bullet 373$ learning activities and 104 assessment questions directed to the study of the astronomy in Obligatory Education, provided by 14 teachers ( 7 from primary education and 7 from secondary education), are analyzed. This analysis aims to elucidate, which conceptual aspects and which cognitive-linguistic skills are included in the activities. Results show that teachers tend to prioritize the study of the most complex aspects such as the Earth and the space. Regarding the cognitive-linguistic skills, teachers tend to demand theoretical models' descriptions. Furthermore, an unbalance between these skills and the study of astronomic issues is observed. Didactic recommendations derived from our results are proposed.

KEY WORDS: astronomy; cognitive-linguistic skills; activities; primary and secondary teachers.

Fecha de recepción: enero 2014 • Aceptado: enero 2015

González-Rodríguez, C., García-Barros, S., Martínez, C. (2015) Qué contenidos y qué habilidades cognitivo-lingüísticas emplea el profesorado de primaria y secundaria en la enseñanza de la astronomía. Enseñanza de las Ciencias, 33.2, pp. 71-89 


\section{INTRODUCCIÓN}

A lo largo de los últimos años en la enseñanza de las ciencias se ha llegado a un consenso bastante amplio sobre qué enseñar. Así, la ciencia escolar plantea como objetivo general que los futuros ciudadanos conozcan los fenómenos del mundo, traten de entenderlos e interpretarlos empleando modelos teóricos, y desarrollen comportamientos adecuados y responsables en la sociedad. De este modo, la educación científica atiende al conocimiento, pero también a la acción (Izquierdo y Adúriz Bravo, 2003; Izquierdo y Aliberas, 2004). Paralelamente, en el marco de la enseñanza de las ciencias se viene defendiendo la necesidad de potenciar el lenguaje oral y escrito, pues favorece el aprendizaje, en la medida que permite expresar y organizar ideas haciendo que el lenguaje cotidiano evolucione hacia aquel que demanda la ciencia escolar (Izquierdo y Aliberas, 2004; Jimenez Alexandre, 2003, 2010; Prain, 2006; Sutton, 2003). Lo indicado resulta coherente con las actuales tendencias curriculares que conciben las competencias como los entes organizadores del currículum (véase LOE, 6 de mayo de 2006) (BOE, 4 de mayo de 2006). Las distintas competencias, en la medida en que abarcan saberes, habilidades y actitudes diversas que los sujetos deben poner en práctica de forma integrada en contextos y situaciones diversas (Cañas, Martin-Díaz y Nieda, 2007), se potencian mutuamente en el marco de las diferentes asignaturas. En este sentido, la interrelación entre la competencia científica y la lingüística ha sido ampliamente defendida desde ambos campos del conocimiento (Pérez Esteve y Zayas, 2007). Desde esta perspectiva es muy significativa la asociación que realiza Jorba (Jorba, 2000) entre la comunicación, producida mediante diferentes tipologías textuales, y las habilidades cognitivas que se activan, refiriéndose así a las habilidades cognitivo-lingüísticas. De esta forma habilidades cognitivas y cognitivo-lingüísticas se potencian entre sí. Con relación a estas últimas se han identificado distintos tipos cuya dificultad y exigencia cognitiva es diferente. Así, por ejemplo, describir consiste en enumerar cualidades, propiedades, etc., de objetos, hechos o fenómenos observables o incluso de modelos abstractos (modelo corpuscular de la materia, modelo de célula, etc.); definir implica expresar las características esenciales de un concepto, con objeto de que no se pueda confundir con otro; justificar supone la expresión de una interpretación de hechos o fenómenos empleando un marco teórico.

Las habilidades en general (De Pro, 1998) y las cognitivo-lingüísticas en particular (Sanmartí, 2007) han de enseñarse de forma explícita en el aula a través de actividades específicas, siendo el profesorado el último responsable de ello. Sin embargo, y a pesar de su importancia, desde la enseñanza de las ciencias no se desarrollan suficientemente.

La calidad de la enseńanza depende muy directamente de la profesionalidad del docente y de su capacidad de innovación, que se halla directamente condicionada por las concepciones personales, influidas por su propia cultura y actividad profesional, vivencias, etc. (Mellado, 2001; Porlan et al., 2010). Por lo tanto, el conocimiento de cómo actúa el profesorado, tanto novel como veterano, cuáles son sus concepciones profesionales, científicas, etc., constituye un punto clave al que atiende la investigación con la intención de mejorar la calidad de la educación científica. Concretamente en lo que respecta a las habilidades cognitivo-lingüísticas se realizaron estudios dirigidos a identificar cuáles de ellas exige el profesorado universitario en las actividades de enseńanza utilizadas en la carrera de Psicopedagogía (Vázquez et al., 2008) o en las actividades dirigidas a la formación de maestros de primaria en materias de Didáctica de las Ciencias (Couto Candedo, García Barros y Martínez Losada, 2013). En el primero se detecta una mayor presencia de la definición y la descripción que de la justificación y la argumentación, mientras que en el segundo se denuncia la baja presencia de esta última. Otros estudios analizan las habilidades cognitivo-lingüísticas implicadas en textos elaborados por profesores de química en formación y su relación con la conceptualización de aspectos relativos a la termodinámica (Ospina Quintero y Bonan, 2011). Así mismo se ha analizado la capacidad del profesorado de 
primaria y secundaria en formación para incluir los distintos tipos de habilidades cognitivo-lingüísticas en las cuestiones de evaluación sobre la nutrición humana (Martínez Losada y García Barros, 2013).

En un estudio precedente hemos relacionado las habilidades cognitivo-lingüísticas con la astronomía (García Barros y Martínez Losada, 2014). Este tema encierra un importante interés educativo, posee una presencia curricular continuada a lo largo de la educación primaria (DOG, 13 de julio de 2007) y primer ciclo de secundaria obligatoria (DOG, 9 de julio de 2007), y suscita problemas de enseñanza/ aprendizaje atendidos por la investigación, como por ejemplo la detección de concepciones alternativas en niños y jóvenes (Baxter, 1989; De Manuel y Graus, 1996; Nussbaum, 1979; Palomar, 2014; Plummer, 2009; Vosniadou y Brewer, 1992) y también en adultos y profesores (Bell y Trundle, 2008; Bryce y Blown, 2012), o el diseño y evaluación de propuestas de enseñanza dirigidas a escolares (Plummer, WasKoa y Slagleb, 2011), a profesores en formación (Bell, 2008; García Barros, Mondelo y Martínez Losada, 1996; Navarrete, 2003; Trumper, 2006) y en ejercicio (Vega Navarro, 2001; Shen y Confrey, 2007, 2010). Sin embargo, la relación entre astronomía y lenguaje ha recibido menos consideración (Lelliott y Rollnick, 2010), a pesar de la importancia que tiene la elaboración de textos y discursos tanto descriptivos como justificativos en el aprendizaje de las regularidades astronómicas y su explicación teórica a través de modelos. En nuestro estudio (García Barros y Martínez Losada, 2014), que emplea un cuestionario abierto/cerrado como instrumento de recogida de datos, se muestra cómo valoran un total de 113 profesores en ejercicio de primaria y secundaria las distintas habilidades cognitivo-lingüísticas asociadas a aspectos concretos (cambios astronómicos diarios, mensuales y anuales, y su explicación). Se aprecia que ambos colectivos otorgan más relevancia a la definición y a la descripción de los modelos teóricos (rotación/traslación terrestre) que a la descripción de fenómenos observables, cuya importancia ha sido destacada por distintos autores (Gavidia, 2014; Navarro, 2011; Plummer, Waskoa y Slagleb, 2011). La descripción y el consecuente conocimiento de los fenómenos observables sirve de base para avanzar en la identificación de cambios y regularidades astronómicas, así como para justificar cuál es la causa de los movimientos aparentes de los astros, usando el modelo teórico que toma así su verdadero sentido (Navarro, 2011; Shen y Confrey, 2010). Por ello es deseable que en la enseñanza del contenido astronómico se promueva el uso equilibrado de las distintas habilidades cognitivo-lingüísticas.

Tomando los resultados del citado estudio como referente, en este trabajo pretendemos profundizar en el pensamiento de un grupo reducido de profesores en ejercicio de educación primaria y secundaria, acercándonos a sus decisiones curriculares. Para ello analizaremos sus actividades de aula y las cuestiones que incluyen en las pruebas de evaluación. Ambas implican necesariamente la producción de textos por parte de los alumnos, lo que nos permitirá conocer en qué habilidades cognitivo-lingüísticas están insistiendo los docentes. Además, podremos conocer los contenidos astronómicos a los que dan importancia. Más concretamente trataremos de dar respuesta a las siguientes cuestiones:

- ¿Qué aspectos sobre astronomía trabaja un pequeño grupo de profesores de primaria y de secundaria en las actividades de enseñanza y cuáles evalúan? ¿Qué habilidades cognitivo-lingüísticas exigen en ellas?

- ¿Se establece algún tipo de asociación entre el uso de habilidades cognitivo-lingüísticas y aspectos astronómicos concretos? ¿Se trabajan estas habilidades de forma equilibrada en relación con dichos aspectos?

\section{METODOLOGÍA}

Han participado 14 profesores: 7 impartían docencia en el último ciclo de educación primaria ( 5 de ellos tenían más de 15 años de experiencia en los distintos ciclos de primaria) y 7 impartían docencia en el primer ciclo de ESO con experiencia superior a 15 años en este ciclo. La colaboración 
fue voluntaria, en respuesta a una solicitud realizada a la dirección de los centros educativos de la zona metropolitana de A Coruña, evitándose la participación de más de un docente por centro.

Los profesores se identificaron como Pp1, Pp2, .. Pp7 los de primaria y como Ps1, Ps2, ... Ps7 los de secundaria. Cada uno aportó entre 5 y 52 actividades (Ac.) del libro de texto habitual y/o de otro origen, que utilizaba con sus alumnos en el tema de astronomía en el curso en que participó en este estudio. Estas actividades eran de lápiz y papel y se realizaban de forma individual o en pequeño grupo. Todos los participantes excepto tres (Ps2, Ps3 y Ps5) empleaban libro de texto que correspondía a las siguientes editoriales: Anaya (Pp1, Pp5 y Ps7), Oxford (Ps1 y Ps6), Vicens Vives (Pp3 y Ps4), Edebe (Pp4 y Pp6), SM (Pp2) y Santillana (Pp7). Además, todos, excepto Pp7, facilitaron las cuestiones de evaluación (Ev.) empleadas en ese curso académico, oscilando su número entre 4 y 17 preguntas. Se analizaron un total de 373 actividades y 103 cuestiones de evaluación. En la tabla 1 se recoge el número que aporta cada docente.

Tabla 1.

Número de actividades (Ac.) y cuestiones de evaluación (Ev.) que emplea cada profesor en el tema de astronomía

\begin{tabular}{|c|c|c|c|c|c|c|c|c|c|c|c|c|c|c|c|}
\hline & \multicolumn{7}{|c|}{ Profesores de primaria } & \multicolumn{7}{|c|}{ Profesores de secundaria } & \multirow{2}{*}{ Total } \\
\hline & Pp1 & $\mathrm{Pp} 2$ & Pp3 & Pp4 & Pp5 & Pp6 & Pp7 & Ps1 & Ps2 & Ps3 & Ps4 & Ps5 & Ps6 & Ps7 & \\
\hline Ac. & 21 & 14 & 5 & 20 & 18 & 24 & 20 & 43 & 24 & 21 & 38 & 32 & 41 & 52 & 373 \\
\hline Ev. & 6 & 4 & 5 & 5 & 10 & 10 & 0 & 4 & 6 & 8 & 12 & 17 & 12 & 4 & 103 \\
\hline
\end{tabular}

El análisis de las actividades y preguntas de evaluación se centró en el aspecto teórico que concretamente se trata en estas. Así, se establecieron cuatro categorías referidas al estudio de: $a$ ) los cambios astronómicos diarios; b) los cambios astronómicos mensuales -estudio de la Luna-; c) los cambios astronómicos anuales, y d) la Tierra en el espacio - planetas del sistema solar, galaxias, etc.-. Estos ámbitos son los que abarca en general el currículum oficial (LOE) respecto a la astronomía.

También se analizaron las habilidades cognitivo-lingüísticas que demandan las actividades/cuestiones de evaluación, en relación con cada uno de los aspectos tratados. Se consideraron cuatro habilidades: a) descripción de un fenómeno observable (D.H/F); b) descripción de un modelo teórico (D.M.); c) definición (Dfi.), y d) justificación de hechos/fenómenos (J.). En las tres primeras se contemplaron dos posibilidades: a) que la exigencia comunicativa fuera directa, es decir, que se solicite al alumno/a una definición o una descripción, considerando como tal la realización de un dibujo/esquema, etc., y b) que la exigencia comunicativa sea indirecta, al no existir tal exigencia en sentido estricto, es decir, el alumno debe disponer del conocimiento/habilidad para responder, pero no se le demanda específicamente la elaboración de un texto más o menos amplio, sino el uso de una palabra, la identificación de datos o de frases verdadero/falso, etc. En el caso de la justificación solo se contempló la exigencia directa porque, en las actividades y cuestiones de evaluación analizadas, esta habilidad siempre ha requerido la elaboración de un texto más o menos amplio por parte del alumno. En este trabajo, y a pesar de su importancia, no se ha considerado la habilidad cognitivo-lingüística "argumentación» porque en ninguna de las actividades y cuestiones de evaluación se solicitaba explícitamente, es decir, en ninguna se demandaba una «razón justificada» aportando datos para convencer a otro.

En la tabla 2 se presentan ejemplos ilustrativos de cuestiones incluidas en las actividades y preguntas de evaluación correspondientes a cada una de las habilidades cognitivo-lingüísticas empleadas. En «otras» se recogen aquellas que no pueden incluirse en las anteriores, pues solicitan otro tipo de habilidades, como exponer/hallar medidas de tiempo, tamaños y distancias. Conviene indicar que en las actividades y/o en las preguntas de evaluación, puede tratarse más de un aspecto astronómico o más de un tipo de habilidad cognitivo-lingüística. 
Cada actividad y cuestión de evaluación fue analizada independientemente por al menos dos autoras/es, que discutieron las posibles discrepancias entre el equipo, que no llegaron a superar el 5\%.

Tabla 2 .

Ejemplos de enunciados de actividades y cuestiones de evaluación que demandan los distintos tipos de habilidades cognitivo-lingüísticas considerados en este estudio

\begin{tabular}{|c|c|c|}
\hline \multicolumn{2}{|c|}{ Habilidades cognitivolingüísticas } & Ejemplos \\
\hline \multirow{2}{*}{$\begin{array}{l}\text { D.H/F Descripción } \\
\text { de un hecho o fenó- } \\
\text { meno observable }\end{array}$} & Directa & $\begin{array}{l}\text { Actividad: } \\
\text { ¿Cómo es la superficie de la Luna? (Ps3) } \\
\text { Describe un eclipse (Pp1) } \\
\text { Cuestión de evaluación: } \\
\quad \text { Nombra y dibuja las fases de la Luna (Pp5) }\end{array}$ \\
\hline & Indirecta & $\begin{array}{l}\text { Actividad: } \\
\text { Se presentan dibujos de la Luna en distintas fases... Se pide: Nombrar la fase que } \\
\text { corresponde y la secuencia de acuerdo con la secuencia natural (Pp2) } \\
\text { Cuestión de evaluación: } \\
\text { Se facilitan tres dibujos, en cada uno se observa un Sol, una persona y la sombra } \\
\text { que proyecta. Indica a qué momento del día corresponde cada dibujo (Ps6) }\end{array}$ \\
\hline \multirow{2}{*}{$\begin{array}{l}\text { D.M. Descripción } \\
\text { de un modelo/hecho } \\
\text { no observable }\end{array}$} & Directa & $\begin{array}{l}\text { Actividad: } \\
\text { ¿Qué movimiento realiza la Luna y alrededor de qué astro? (Ps4) } \\
\text { Se propone una dramatización en la que cada niño/a va a desempeñar un papel } \\
\text { (Sol, Tierra...) y deberá realizar el movimiento correcto según lo que se le pida en } \\
\text { cada momento (Pp7) } \\
\text { Cuestión de evaluación: } \\
\text { ¿Qué movimientos realiza la Tierra en el espacio? (Ps2) }\end{array}$ \\
\hline & Indirecta & $\begin{array}{l}\text { Actividad: } \\
\text { El planeta más grande del sistema solar es... El planeta del sistema solar más perecido } \\
\text { a la Tierra es... (Ps3) } \\
\text { Cuestión de evaluación: } \\
\text { ¿Qué movimiento se representa en cada dibujo y qué consecuencias tiene (Pp2) }\end{array}$ \\
\hline \multirow[t]{2}{*}{$D f i$. Definición } & Directa & $\begin{array}{l}\text { Actividad: } \\
\text { ¿En qué consiste la fusión nuclear? (Ps1) } \\
\text { ¿Qué es una galaxia? (Ps4) } \\
\text { Define: movimiento de rotación de la Tierra (Pp1) } \\
\text { Cuestión de evaluación: } \\
\text { ¿Qué son los planetas? (Pp2) }\end{array}$ \\
\hline & Indirecta & $\begin{array}{l}\text { Actividad: } \\
\text { ¿Qué planeta aparece dibujado?, ¿cómo se llaman los otros dos astros que se dibujan? } \\
\text { (Pp1) } \\
\text { Cuestión de evaluación: } \\
\text { Los cúmulos estelares son agrupaciones de planetas (VIF) (Ps3) }\end{array}$ \\
\hline$J$ J Justificación & Directa & $\begin{array}{l}\text { Actividad: } \\
\text { Mercurio y la Luna no tienen atmósfera. ¿Qué consecuencia tiene esto sobre el aspecto } \\
\text { de su superficie? (Ps3) } \\
\text { ¿Por qué se suceden el día y la noche? ¿Y las estaciones del año? (Pp4) } \\
\text { Cuestión de evaluación: } \\
\text { Explica la causa de que haya día y noche (Ps4) }\end{array}$ \\
\hline Otras & & $\begin{array}{l}\text { Actividad: } \\
\text { Realización de un juego donde se resuman aquellas preguntas clave del tema (Pp3) } \\
\text { Calcular el tamaño de los planetas; de distancias en años luz (Ps1) }\end{array}$ \\
\hline
\end{tabular}




\section{RESULTADOS}

\section{Aspectos de astronomía que incluyen los profesores en las actividades y cuestiones de evaluación}

En las actividades dirigidas al estudio de la astronomía, los participantes en este trabajo incluyen los cuatro aspectos considerados (estudio de los cambios astronómicos diarios, mensuales y anuales y el estudio de la Tierra en el espacio) (figura1). Solo dos de ellos omiten alguno: Pp3 no aporta actividades sobre el estudio de los cambios mensuales y anuales y Ps1 no lo hace sobre los cambios diarios.

Las actividades analizadas se dirigen mayoritariamente al estudio de la Tierra en el espacio. Lo indicado es más evidente en el profesorado de secundaria que en el de primaria. Así, todos los docentes de secundaria, excepto Ps6, dirigen más de la mitad de sus actividades a este aspecto, frente a solo tres de primaria (Pp2, Pp3 y $\mathrm{Pp} 6$ ). Los profesores que aportan un número de actividades más equilibrado sobre los diferentes tópicos son Pp5 y Ps6.

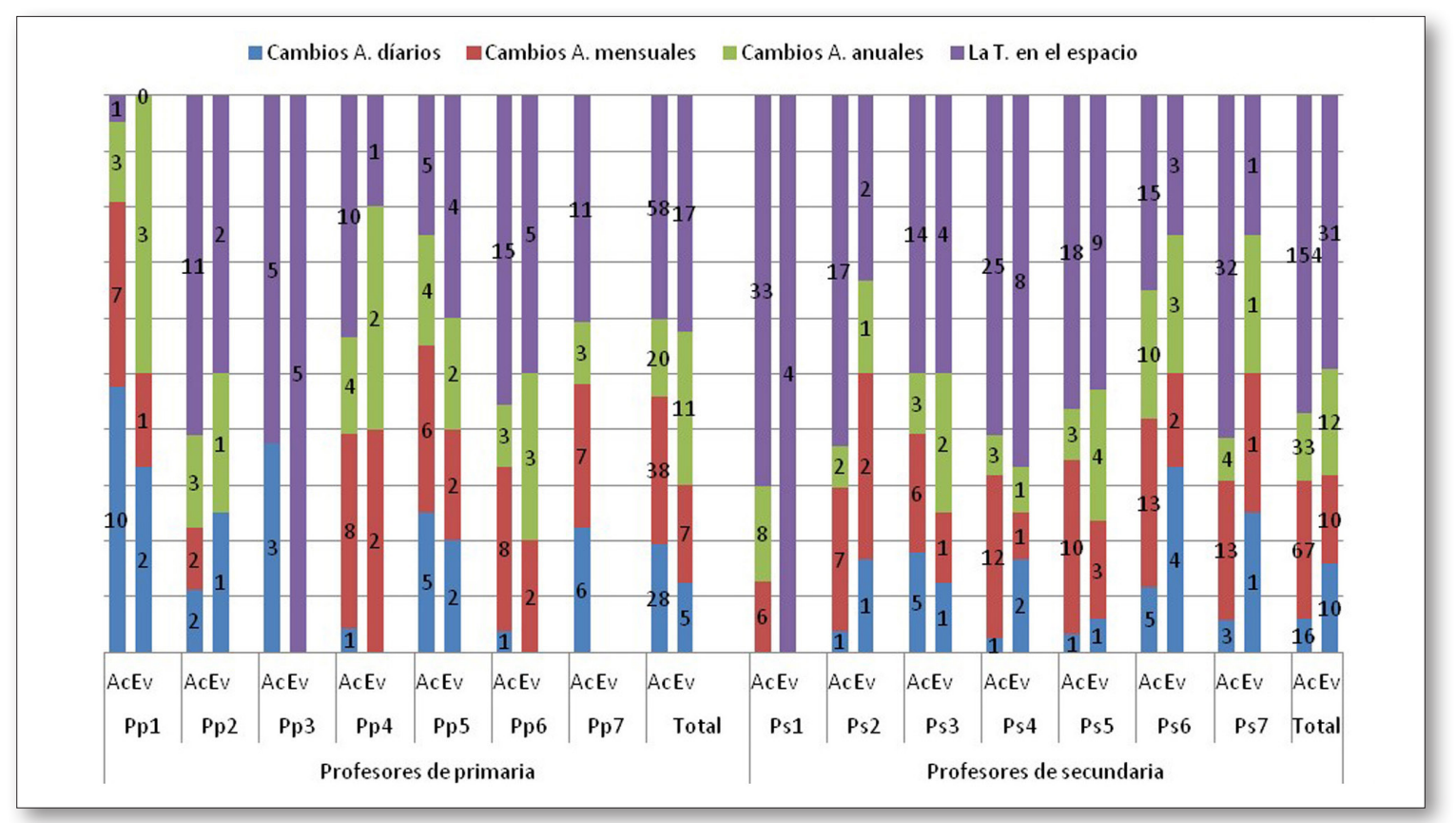

Nota: una misma actividad o cuestión de evaluación puede incluir más de uno de los aspectos astronómicos considerados.

Fig. 1. Aspectos astronómicos tratados por el profesorado de primaria y secundaria en las actividades (Ac.) e incluidos en las cuestiones de evaluación (Ev.).

En las cuestiones de evaluación (figura 1), los participantes de primaria generalmente plantean preguntas sobre tres aspectos astronómicos; solo $\mathrm{Pp} 5$ aportó cuestiones correspondientes a los cuatro aspectos, mientras que Pp3 se centró en uno -la Tierra en el espacio-. Este tópico y el estudio de los cambios anuales son los más demandados en la evaluación, pues lo incluyen en ella cinco de los seis profesores, mientras que los cambios mensuales y diarios se evalúan en menor medida; únicamente cuatro y tres docentes respectivamente.

Todos los participantes de secundaria han seleccionado preguntas de evaluación de todos los aspectos, excepto Ps1, que solo aportó cuestiones sobre la Tierra en el espacio. Este aspecto también ha sido el priorizado por otros tres docentes (Ps3, Ps4 y Ps5), que dirigen a este la mitad o más de sus preguntas de evaluación. Los demás profesores evalúan los distintos tópicos de forma más equilibrada. 


\section{Habilidades cognitivo-lingüísticas que exigen los profesores en las actividades y cuestio- nes de evaluación}

En sus actividades, los participantes exigen distintos tipos de habilidades cognitivo-lingüísticas, aunque algunos de ellos también demandan habilidades agrupadas bajo el epígrafe de «otras» (figura 2). De los colaboradores de primaria, cuatro exigen los cuatro tipos de habilidades, mientras que el resto omiten alguna de ellas: la justificación (Pp2 y Pp3), la descripción de fenómenos (Pp3) y la definición (Pp7). Además, se aprecia que este colectivo tiende a priorizar la descripción de modelos. Tres profesores (Pp2, Pp4 y Pp6) la solicitan en más de la mitad de las actividades aportadas y otros dos (Pp3 y $\mathrm{Pp} 5$ ) la priorizan junto a otra habilidad (definición y descripción de fenómenos respectivamente). Cabe destacar que solo un docente (Pp1) prioriza la justificación.

Todos los colaboradores de secundaria, menos uno, incluyen los cuatro tipos de habilidades en sus actividades, priorizando también, excepto Ps3, la descripción de modelos. Así, tres profesores (Ps1, Ps2 y Ps4) la requieren en la mitad o más de sus actividades y dos (Ps5 y Ps6) lo hacen junto a otra habilidad (descripción de fenómenos y justificación respectivamente). Ps7 trata equilibradamente todas las habilidades excepto la justificación que comparativamente minimiza. Tal minimización se aprecia también en otros cuatro participantes, llegando uno de ellos (Ps2) a omitirla.

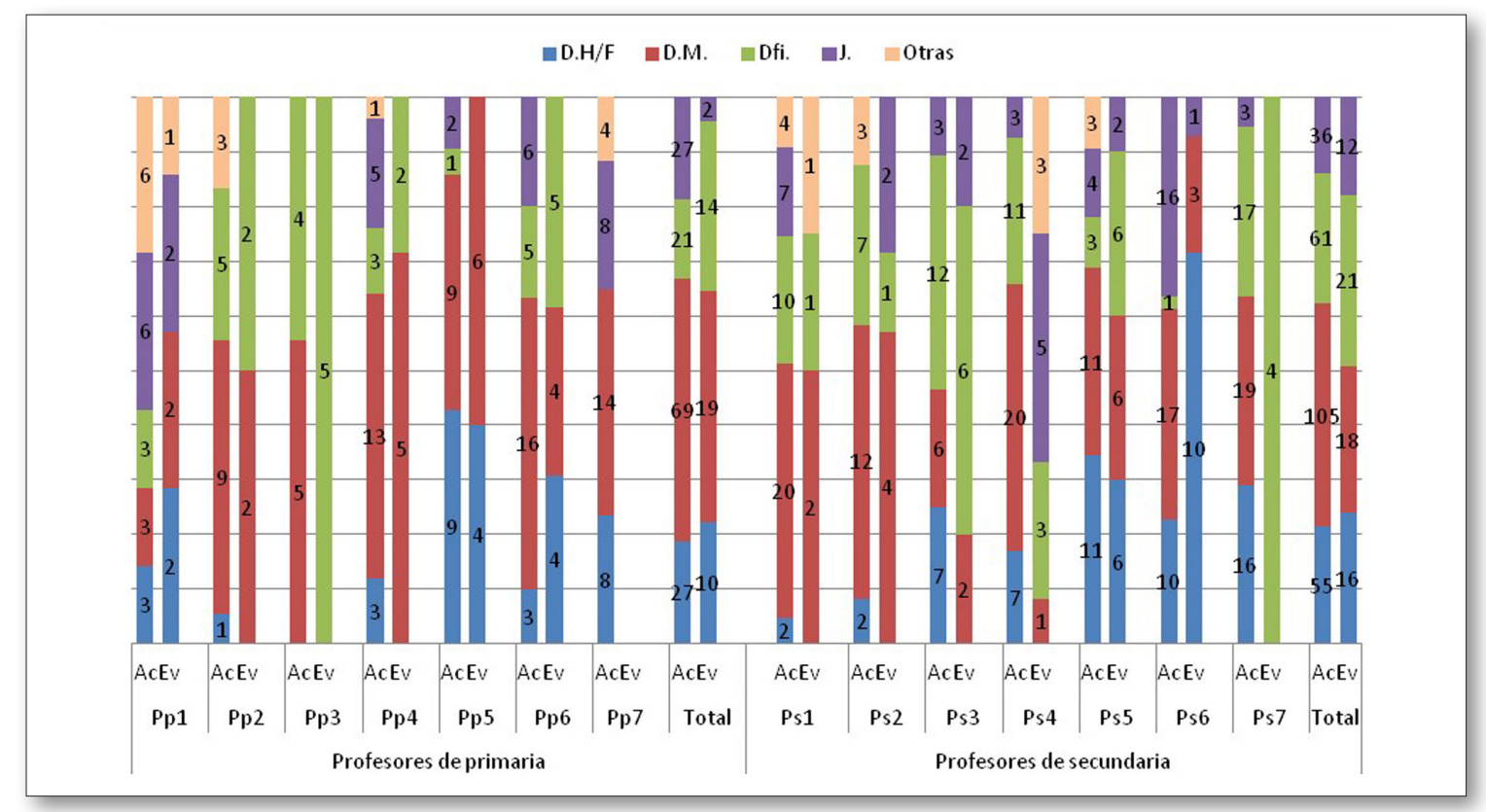

Nota: una misma actividad o cuestión de evaluación puede exigir más de una habilidad cognitivo-lingüística.

Fig. 2. Habilidades cognitivo-lingüísticas (descripción de hechos/fenómenos -D.H/F-, descripción de modelos teóricos -D.M-, definición -Dfi.-, justificación -J.- y otras), exigidas en las actividades (Ac.) y cuestiones de evaluación (Ev.) que emplea el profesorado de primaria y secundaria en el tema de astronomía.

En las cuestiones de evaluación, las habilidades que demandan ambos grupos de profesores son menos variadas que las propuestas en las actividades (figura 2). Así, ningún profesor de primaria exige las cuatro habilidades consideradas, dos de ellos incluyen tres tipos de habilidades y los demás dos o incluso una. Al igual que en las actividades, la habilidad con más presencia en la evaluación es la descripción de modelos, pues la solicitan todos los participantes, excepto Pp3, que solo evalúa la definición. Esta habilidad es requerida por otros profesores (Pp2, Pp4 y Pp6), lo mismo que la descripción de hechos/ fenómenos (Pp1, Pp5 y Pp6), mientras que la justificación solo la exige Pp1. 
Solo un profesor de secundaria demanda todas las habilidades en sus cuestiones de evaluación, los demás solicitan tres, dos o incluso una. Igualmente, la descripción de modelos es la habilidad más solicitada por todo el profesorado de secundaria, aunque la definición y la justificación también tienen una presencia importante. En concreto, la definición la contemplan todos los profesores excepto Ps6 y la justificación todos excepto Ps1 y Ps7. Sin embargo la descripción de hechos/fenómenos solamente la demandan dos docentes (Ps5 y Ps6).

A continuación, profundizamos en el análisis de las tres habilidades cognitivo-lingüísticas (la descripción de hechos/fenómenos, la descripción de modelos y la definición) que se pueden requerir de forma directa o indirecta en las actividades y cuestiones de evaluación En cómputos totales, el profesorado participante de primaria solicita en mayor medida la forma indirecta en las actividades que propone. Lo mismo ocurre con las cuestiones de evaluación, excepto en el caso de la definición, donde la forma directa es superior (tabla 3). Además, cuatro docentes exigen ciertas habilidades de una única manera, siendo la forma indirecta la solicitada por todos ellos, excepto por Pp3, que exige la definición solo de forma directa en sus cuestiones de evaluación.

Tabla 3.

Número de actividades/cuestiones de evaluación empleadas por los docentes de primaria en las que se trabaja cada habilidad directa (Dir.) e indirectamente (Ind.)

\begin{tabular}{|c|c|c|c|c|c|c|c|c|c|c|c|c|c|c|c|c|c|}
\hline \multirow{2}{*}{\multicolumn{2}{|c|}{$\begin{array}{c}\text { Habilidades } \\
\text { cognitivo-lingüísticas }\end{array}$}} & \multicolumn{2}{|c|}{ Pp1 } & \multicolumn{2}{|c|}{ Pp2 } & \multicolumn{2}{|c|}{ Pp3 } & \multicolumn{2}{|c|}{ Pp4 } & \multicolumn{2}{|c|}{ Pp5 } & \multicolumn{2}{|c|}{ Pp6 } & \multicolumn{2}{|c|}{ Pp7 } & \multicolumn{2}{|c|}{ Total } \\
\hline & & $\mathrm{Ac}_{\mathrm{c}}$ & $\mathrm{Ey}$ & $A_{c}$ & Ey & $A_{c}$ & Ey & $\mathrm{Ac}_{\mathrm{C}}$ & $\mathrm{Ey}$ & $A_{c}$ & Ey & $A_{c}$ & Ey & $A_{c}$ & Ey & $A_{c}$ & Ey \\
\hline \multirow{2}{*}{ D. H/F } & Dir. & 1 & - & - & - & - & - & 2 & - & 4 & 3 & 2 & 2 & 2 & - & 11 & 5 \\
\hline & Ind. & 1 & 2 & 1 & & & - & 1 & - & 4 & 2 & 1 & 2 & 6 & - & 14 & 6 \\
\hline \multirow{2}{*}{ D.M. } & Dir. & 2 & - & 6 & 2 & 1 & - & 4 & 1 & 5 & 2 & 7 & 3 & 6 & - & 31 & 8 \\
\hline & Ind. & 1 & 1 & 3 & 1 & 4 & - & 9 & 4 & 4 & 4 & 9 & 1 & 8 & - & 38 & 11 \\
\hline \multirow{2}{*}{$D f i}$. & Dir. & 3 & - & - & - & 3 & 5 & 1 & 1 & - & - & 3 & 3 & - & - & 10 & 9 \\
\hline & Ind. & 1 & - & 5 & 1 & 1 & - & 2 & 1 & 1 & - & 2 & 2 & - & - & 12 & 4 \\
\hline
\end{tabular}

Nota: una misma actividad o cuestión de evaluación puede exigir más de una habilidad cognitivo-lingüística.

Los participantes de secundaria, tanto en las actividades como en las cuestiones de evaluación, en cómputos totales demandan la definición más directa que indirectamente, ocurriendo lo contrario con la descripción de hechos/fenómenos. La descripción de modelos se requiere de ambas formas casi por igual en actividades, y directamente en mayor proporción en las preguntas de evaluación (tabla 4). 
Tabla 4 .

Número de actividades/cuestiones de evaluación empleadas por los docentes de secundaria en las que se trabaja cada habilidad directa (Dir.) e indirectamente (Ind.)

\begin{tabular}{|c|c|c|c|c|c|c|c|c|c|c|c|c|c|c|c|c|c|}
\hline \multirow{2}{*}{\multicolumn{2}{|c|}{$\begin{array}{c}\text { Habilidades } \\
\text { cognitivo-lingüísticas }\end{array}$}} & \multicolumn{2}{|c|}{ Ps 1} & \multicolumn{2}{|c|}{ Ps2 } & \multicolumn{2}{|c|}{ Ps3 } & \multicolumn{2}{|c|}{ Ps4 } & \multicolumn{2}{|c|}{ Ps 5} & \multicolumn{2}{|c|}{ Ps6 } & \multicolumn{2}{|c|}{ Ps7 } & \multicolumn{2}{|c|}{ Total } \\
\hline & & Ac. & Ev. & Ac. & Ev. & Ac. & Ev. & Ac. & Ev. & Ac. & Ev. & Ac. & Ev. & Ac. & Ev. & Ac. & Ev. \\
\hline \multirow{2}{*}{ D.H/F } & Dir. & - & - & 1 & - & 1 & - & 4 & - & 5 & 1 & 2 & 3 & 6 & - & 19 & 4 \\
\hline & Ind. & 2 & - & 1 & - & 6 & - & 3 & - & 6 & 8 & 8 & 7 & 10 & - & 36 & 15 \\
\hline \multirow{2}{*}{ D.M. } & Dir. & 16 & 2 & 7 & 4 & 3 & 2 & 6 & - & 5 & 8 & 4 & - & 9 & - & 50 & 16 \\
\hline & Ind. & 4 & - & 5 & - & 3 & - & 14 & 1 & 7 & 4 & 10 & - & 10 & - & 53 & 5 \\
\hline \multirow{2}{*}{$D f i}$. & Dir. & 9 & 1 & 7 & 1 & 5 & 3 & 6 & - & 2 & 6 & - & - & 10 & 4 & 39 & 15 \\
\hline & Ind. & 1 & - & - & - & 7 & 3 & 5 & 3 & 2 & 1 & - & - & 7 & - & 22 & 7 \\
\hline
\end{tabular}

Nota: una misma actividad o cuestión de evaluación puede exigir más de una habilidad cognitivo-lingüística.

Por otra parte cabe destacar que cinco docentes de secundaria solicitan algunas de las habilidades únicamente de una forma, siendo la directa más habitual. Así, en evaluación cuatro docentes (Ps1, Ps2, Ps3 y Ps7) demandan solo directamente la descripción de modelos y/o la definición; esta también es requerida solo directamente por Ps2 en actividades.

\section{Asociaciones que establecen los profesores entre habilidades cognitivo-lingüísticas y aspec- tos astronómicos concretos}

Más de la mitad de los profesores de primaria de este estudio trabajan conjuntamente en sus actividades: a) la descripción de modelos en relación con todos los tópicos astronómicos considerados; $b$ ) la justificación asociada al estudio de los cambios astronómicos y a la Tierra en el espacio; $c$ ) la descripción de hechos/fenómenos en relación con los cambios mensuales, y d) la definición en relación con la Tierra en el espacio. Las otras asociaciones posibles fueron minoritarias. Por otra parte, en las cuestiones de evaluación, más de la mitad de los profesores de primaria relacionan: a) la descripción de modelos concretamente con los cambios astronómicos diarios y anuales y b) la definición con el estudio de la Tierra en el espacio. Las otras asociaciones posibles fueron minoritarias (tabla 5). 
Tabla5.

Profesores de primaria que en actividades y/o cuestiones de evaluación relacionan las distintas habilidades cognitivo-lingüísticas con los aspectos de astronomía considerados

\begin{tabular}{|c|c|c|c|c|c|c|c|c|}
\hline \multirow{2}{*}{$\begin{array}{l}\text { Habilidades } \\
\text { cognitivo- } \\
\text { lingüísticas }\end{array}$} & \multicolumn{2}{|c|}{$\begin{array}{l}\text { C. astronómicos diarios } \\
\text { N. }{ }^{\circ} \text { prof }=7\end{array}$} & \multicolumn{2}{|c|}{$\begin{array}{l}\text { C. astronómicos } \\
\text { mensuales } \\
\text { N. }{ }^{\circ} \text { prof }=6\end{array}$} & \multicolumn{2}{|c|}{$\begin{array}{l}\text { C. astronómicos } \\
\text { anuales } \\
\text { N. }{ }^{\circ} \text { prof }=6\end{array}$} & \multicolumn{2}{|c|}{$\begin{array}{c}\text { La Tierra } \\
\text { en el espacio } \\
\text { N.o prof }=6\end{array}$} \\
\hline & Ac. & Ev. & Ac. & Ev. & Ac. & Ev. & Ac. & Ev. \\
\hline D. $H / F$ & $\begin{array}{c}\text { Pp1 Pp5 } \\
\text { Pp7 } \\
\text { n=3 }\end{array}$ & & $\begin{array}{c}\text { Pp1 Pp4 } \\
\text { Pp5 Pp6 } \\
\text { Pp7 } \\
\text { n=5 }\end{array}$ & $\begin{array}{c}\text { Pp1 Pp6 } \\
\text { n=2 }\end{array}$ & Pp5 & $\begin{array}{c}\text { Pp1 } 1^{*} \mathrm{Pp} 5 \\
\text { Pp6* } \\
\text { n=3 }\end{array}$ & $\begin{array}{c}\text { Pp2 Pp7 } \\
n=2\end{array}$ & \\
\hline D.M. & $\begin{array}{c}\text { Pp1 Pp2 } \\
\text { Pp3 Pp5 } \\
\text { Pp7 } \\
\text { n=5 }\end{array}$ & $\begin{array}{l}\text { Pp1 Pp2 } \\
\text { Pp5 Pp7 } \\
\text { n=4 }\end{array}$ & $\begin{array}{l}\text { Pp1 Pp4 } \\
\text { Pp5 Pp6 } \\
\text { Pp7 } \\
\text { n=5 }\end{array}$ & $\begin{array}{l}\text { Pp4 } \\
n=1\end{array}$ & $\begin{array}{l}\text { Pp2 Pp4 } \\
\text { Pp5 Pp6 } \\
\text { Pp7 } \\
\text { n=5 }\end{array}$ & $\begin{array}{c}\text { Pp2 Pp4 } \\
\text { Pp5 Pp6 } \\
\text { n=4 }\end{array}$ & $\begin{array}{c}\text { Pp2 Pp3 } \\
\text { Pp4Pp5 } \\
\text { Pp6 Pp7 } \\
n=6\end{array}$ & P5 P6 \\
\hline$D f$. & $\begin{array}{l}\text { Pp1 Pp3 } \\
\text { n=2 }\end{array}$ & & $\begin{array}{l}\mathrm{Pp} 2 \\
\mathrm{n}=1\end{array}$ & & $\begin{array}{c}\text { Pp1 Pp2 } \\
\text { n=1 }\end{array}$ & & $\begin{array}{c}\text { Pp2Pp3 } \\
\text { Pp4 Pp5 } \\
\text { Pp6 } \\
\text { n=5 }\end{array}$ & $\begin{array}{c}\text { Pp2 Pp3 } \\
\text { Pp4 Pp6 } \\
\text { n=4 }\end{array}$ \\
\hline$J$. & $\begin{array}{l}\text { Pp1 Pp4 } \\
\text { Pp5 Pp6 } \\
\text { Pp7 } \\
\text { n=5 }\end{array}$ & $\mathrm{n}=1$ & Pp1 Pp7 & & $\begin{array}{l}\text { Pp1 Pp4 } \\
\text { Pp6 } \\
\text { n=3 }\end{array}$ & $\mathrm{n}=1$ & $\begin{array}{l}\text { Pp1 Pp4 } \\
\text { Pp6 Pp7 } \\
\text { n=4 }\end{array}$ & \\
\hline
\end{tabular}

*Docentes que exigen en evaluación habilidades cognitivo-lingüísticas relativas a un núcleo de estudio que no incluyen en actividades de enseñanza dirigidas a dichos núcleos.

Cabe destacar que, en general, los participantes exigen en la evaluación las mismas habilidades asociadas a los mismos tópicos que también se detectan en sus actividades de enseñanza, aunque Pp1 y Pp6 exigen la descripción de hechos/fenómenos en relación con el estudio astronómico de los cambios anuales únicamente en las cuestiones de evaluación.

Más de la mitad de los profesores participantes de secundaria trabajan en las actividades: a) la descripción de modelos en relación con tres de los tópicos considerados (cambios mensuales, anuales y la Tierra en el espacio); b) la definición asociada a estos mismos tópicos; c) la descripción de hechos/ fenómenos en relación con cambios astronómicos diarios, mensuales y anuales, y d) la justificación en relación con los cambios mensuales y con la Tierra en el espacio. Las otras asociaciones fueron minoritarias. Por otra parte, en sus cuestiones de valuación, más de la mitad de estos profesores establecen las relaciones entre: $a$ ) la descripción de modelos y el estudio de la Tierra en el espacio; $b$ ) la definición y este mismo tópico, y $c$ ) la justificación asociada a los cambios mensuales. Las otras asociaciones posibles fueron minoritarias y una de ellas no detectada (descripción de modelos en relación con el estudio de los cambios mensuales) (tabla 6). 
Tabla 6.

Profesores de secundaria que en actividades y/o cuestiones de evaluación relacionan las distintas habilidades cognitivo-lingüísticas con los aspectos de astronomía considerados

\begin{tabular}{|c|c|c|c|c|c|c|c|c|}
\hline \multirow{2}{*}{$\begin{array}{c}\text { Habilidades } \\
\text { cognitivo- } \\
\text { lingüísticas }\end{array}$} & \multicolumn{2}{|c|}{$\begin{array}{l}\text { C. astronómicos } \\
\text { diarios } \\
\text { N. }{ }^{\circ} \text { prof }=6\end{array}$} & \multicolumn{2}{|c|}{$\begin{array}{c}\text { C. astronómicos } \\
\text { mensuales } \\
\text { N.o prof }=7\end{array}$} & \multicolumn{2}{|c|}{$\begin{array}{l}\text { C. astronómicos } \\
\text { anuales } \\
\text { N. }{ }^{\circ} \text { prof }=7\end{array}$} & \multicolumn{2}{|c|}{$\begin{array}{c}\text { La Tierra } \\
\text { en el espacio } \\
\text { N. }{ }^{\circ} \text { prof }=7\end{array}$} \\
\hline & Ac. & Ev. & Ac. & Ev. & Ac. & Ev. & Ac. & Ev. \\
\hline D. $H / F$ & $\begin{array}{c}\text { Ps3 Ps5 } \\
\text { Ps6 Ps7 } \\
\text { n=4 }\end{array}$ & Ps5 Ps6 & $\begin{array}{c}\text { Ps1 Ps2 Ps3 } \\
\text { Ps4 Ps5 Ps6 } \\
\text { Ps7 } \\
\text { n=7 }\end{array}$ & Ps5 Ps6 & $\begin{array}{c}\text { Ps1 Ps3 Ps5 } \\
\text { Ps6 Ps7 } \\
n=5\end{array}$ & Ps5 Ps6 & $\begin{array}{c}\text { Ps4 Ps5 } \\
\text { Ps7 } \\
\text { n=3 }\end{array}$ & Ps5 \\
\hline D. M. & Ps6 & Ps2* & $\begin{array}{c}\text { Ps1 Ps2 Ps3 } \\
\text { Ps4 Ps5 Ps6 } \\
\text { Ps7 } \\
\mathrm{n}=7\end{array}$ & & $\begin{array}{c}\text { Ps1 Ps2 Ps4 } \\
\text { Ps5 Ps6 Ps7 } \\
n=6\end{array}$ & $\begin{array}{c}\text { Ps2 Ps5 } \\
n=2\end{array}$ & $\begin{array}{c}\text { Ps1 Ps2 Ps3 } \\
\text { Ps4 Ps5 Ps6 } \\
\text { Ps7 } \\
n=7\end{array}$ & $\begin{array}{l}\text { Ps1 Ps2 Ps3 } \\
\text { Ps4 Ps5 Ps6 } \\
n=6\end{array}$ \\
\hline$D f$. & Ps2 & Ps3* Ps7* & $\begin{array}{l}\text { Ps1 Ps2 Ps3 } \\
\text { Ps4 Ps5 Ps7 }\end{array}$ & $\begin{array}{l}\text { Ps3 Ps5 } \\
\text { Ps7 }\end{array}$ & $\begin{array}{l}\text { Ps1 Ps2 Ps3 } \\
\text { Ps5 } \\
\text { Ps7 }\end{array}$ & $\begin{array}{c}\text { Ps3 Ps5 } \\
\text { Ps7 }\end{array}$ & $\begin{array}{c}\text { Ps1 Ps2 Ps3 } \\
\text { Ps4 Ps5 Ps6 } \\
\text { Ps7 }\end{array}$ & $\begin{array}{l}\text { Ps1 Ps2 Ps3 } \\
\text { Ps4 Ps5 Ps7 }\end{array}$ \\
\hline & $\mathrm{n}=1$ & $\mathrm{n}=2$ & $\mathrm{n}=6$ & $\mathrm{n}=3$ & $\mathrm{n}=5$ & $\mathrm{n}=3$ & $\mathrm{n}=7$ & $\mathrm{n}=6$ \\
\hline$J$. & $\begin{array}{c}\text { Ps4 Ps6 } \\
n=2\end{array}$ & Ps4 & $\begin{array}{l}\text { Ps1 Ps3 Ps5 } \\
\text { Ps6 } \\
\\
n=4\end{array}$ & $\begin{array}{l}\text { Ps2* Ps3 } \\
\text { PS4* PS6 } \\
\text { n=4 }\end{array}$ & $\mathrm{n}=1$ & Ps4* & $\begin{array}{l}\text { Ps1 Ps4 Ps5 } \\
\text { Ps6 Ps7 } \\
n=5\end{array}$ & $\begin{array}{l}\text { Ps3* Ps4 } \\
\text { Ps5 } \\
\text { n=3 }\end{array}$ \\
\hline
\end{tabular}

*Docentes que exigen en evaluación habilidades cognitivo-lingüísticas relativas a un núcleo de estudio que no incluyen en actividades de enseñanza dirigidas a dichos núcleos.

Cabe destacar que cuatro profesores exigen habilidades asociadas a determinados tópicos solo en la evaluación sin haberlo hecho en las actividades. Concretamente: a) la descripción de modelos (Ps2) y la definición (Ps3 y Ps7) en relación con el estudio de los cambios diarios, y b) la justificación en relación con el estudio de los cambios mensuales (Ps2 y Ps4), de los cambios anuales (Ps4) y de la Tierra en el espacio (Ps3).

\section{Niveles de adecuación en el uso equilibrado de las habilidades cognitivo-lingüísticas detec- tados en el profesorado participante}

El análisis individualizado de las actividades que emplea cada profesor ha permitido establecer cinco niveles de mayor a menor adecuación. En esta ocasión no se consideraron las cuestiones de evaluación debido al reducido número que emplea alguno de los participantes. La adecuación se basa en la diversidad de habilidades que exigen respecto a los distintos tópicos, contemplándose las tres (descripción de hechos/fenómenos, del modelo y la justificación) que inciden más directamente en el conocimiento de los fenómenos astronómicos y en su interpretación a partir del modelo explicativo:

- Nivel 1. Se tratan las tres habilidades en más de un tópico.

- Nivel 2. Se tratan las tres habilidades en un tópico.

- Nivel 3. Se tratan dos de estas habilidades en más de un tópico.

- Nivel 4. Se tratan dos de estas habilidades en un solo tópico.

- Nivel 5. Se trata una sola habilidad en algún tópico. 
En la figura 3 se visualiza en sombreado qué habilidades trata cada profesor en cada uno de los tópicos y además se identifica en qué nivel de adecuación se sitúa cada uno de los participantes en este estudio.

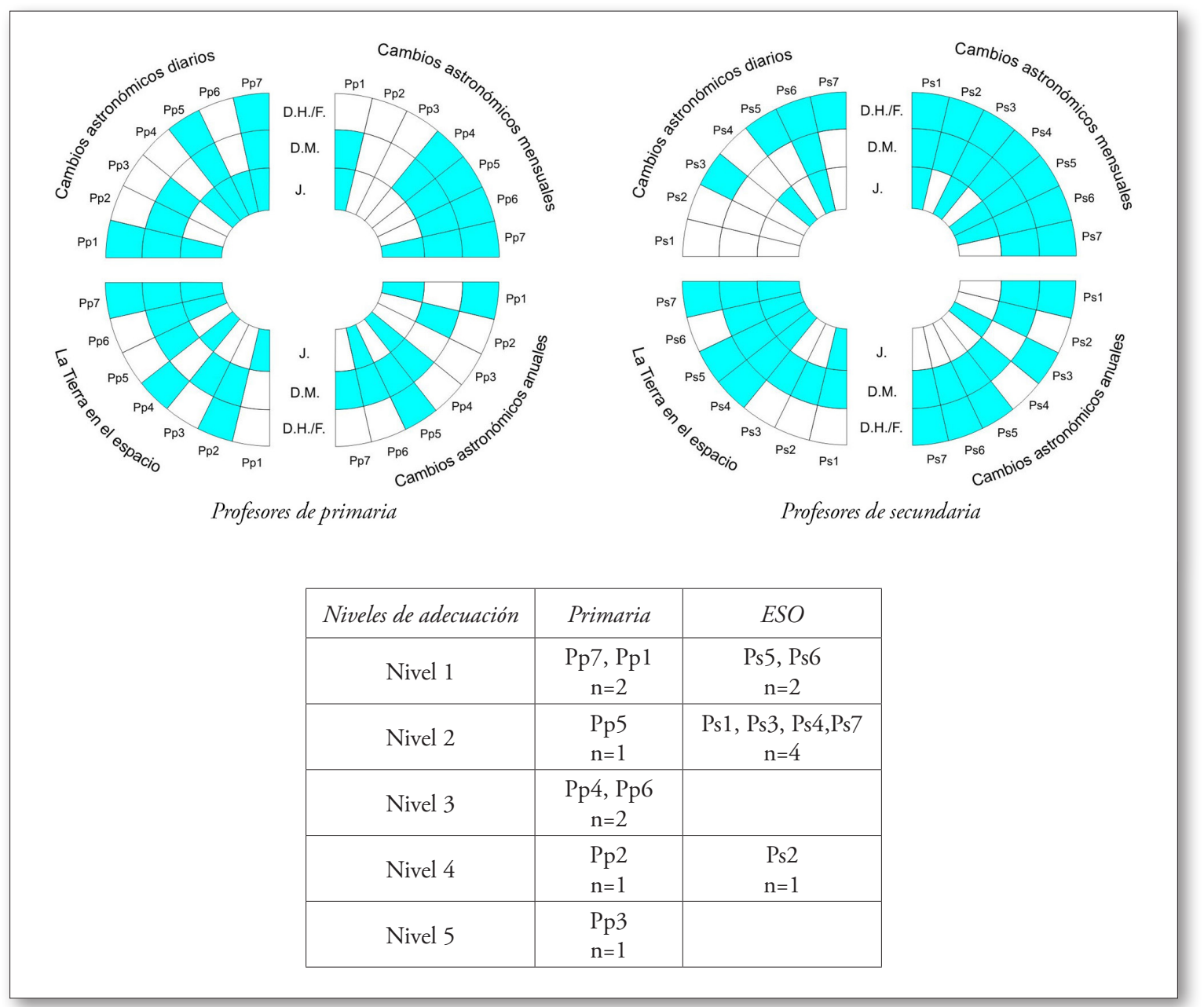

Fig. 3. Habilidades cognitivo-lingüísticas (descripción de hechos/fenómenos -D.H/F.-, descripción de modelos -D.M- y justificación -J.-) que el profesorado incluye en las actividades dirigidas al estudio de los distintos tópicos astronómicos. Niveles de adecuación en los que se sitúa dicho profesorado.

Como se puede apreciar, todos los participantes de secundaria, menos uno, se identifican con los niveles más idóneos (nivel 1 y 2), aunque sobre todo con el nivel 2. Sin embargo, solo tres profesores de primaria se sitúan en los niveles citados, aunque Pp7 es el único que emplea las tres habilidades consideradas en tres tópicos. Cabe destacar que la conjunción de las tres habilidades se halló siempre relacionada con el estudio de los cambios diarios, mensuales y de la Tierra en el espacio. Ninguno de los profesores que alcanza los niveles más idóneos emplea las tres habilidades en relación con el estudio de los cambios astronómicos anuales. 


\section{CONCLUSIONES Y DISCUSIÓN}

En relación con el primer problema -¿Qué aspectos sobre astronomía trabaja un pequeño grupo de profesores de primaria y de secundaria en las actividades de enseñanza y cuáles evalúan? ¿Qué habilidades cognitivo-lingüísticas exigen en ellas?-, se puede afirmar que, en cómputos generales, el profesorado participante, al tratar la astronomía, atiende a los distintos ámbitos considerados, aunque tiende a insistir en aquel más complejo -la Tierra en el espacio-, tema prioritario en actividades y en cuestiones de evaluación. Además, incluso los colaboradores de primaria le dedican proporcionalmente menos actividades al estudio de aspectos astronómicos más próximos y apreciables, lo que pone de manifiesto que no existe un claro progreso entre primaria y secundaria.

En lo que respecta a las habilidades cognitivo-lingüísticas, y también en términos generales, se detecta una preponderancia de la descripción de modelos teóricos (Sol/Tierra; Sol/Tierra/Luna; sistema solar) en actividades y cuestiones de evaluación, siendo la descripción de los fenómenos observables menos frecuente, tanto entre el profesorado de primaria como entre el de secundaria, lo que también nos sugiere la inexistencia de un progreso. Sin embargo, es necesario matizar esta afirmación, porque el profesorado participante de primaria, a pesar de emplear con frecuencia la citada descripción de modelos abstractos, utiliza menos que el de secundaria una habilidad que resulta exigente como es la justificación, que requiere el conocimiento y la utilización del modelo teórico.

En este estudio también se pone de manifiesto que, en diversas actividades utilizadas por los docentes, se exigen las habilidades cognitivo-lingüísticas de forma indirecta, de manera que el alumnado identifica características, nombra las distintas formas de la Luna, identifica los planetas en un esquema del sistema solar, reconoce como verdadera o falsa una definición..., pero no construye exactamente una descripción completa o una definición. Lo indicado es más frecuente entre los colaboradores de primaria que entre los de secundaria, quizá porque sus alumnos/as, más pequeños, tienen mayores dificultades para expresarse. También es más frecuente en las actividades de enseñanza que en la evaluación, quizá porque en esta se exige que el alumnado exprese con más rigor los conocimientos adquiridos, especialmente la definición.

Con relación al segundo problema - ¿Se establece algún tipo de asociación entre el uso de habilidades cognitivo-lingüísticas y aspectos astronómicos concretos? ¿Se trabajan estas habilidades de forma equilibrada en relación con dichos aspectos?-, se aprecia un comportamiento desigual entre los participantes. En este sentido, los docentes de primaria que han colaborado en el trabajo parecen aplicar las habilidades cognitivo-lingüísticas con más intensidad a tópicos concretos (la descripción de los fenómenos observables al ciclo lunar, la definición al estudio del sistema planetario y la justificación a los cambios astronómicos diarios). Por el contrario, el profesorado de secundaria tiende a emplear más variedad de habilidades cognitivo-lingüísticas, asociándolas más o menos por igual a los diferentes tópicos astronómicos. Lo indicado resulta coherente con que sean más los docentes de secundaria los que dispongan de un mejor nivel de adecuación, caracterizado por la utilización equilibrada de las tres habilidades cognitivolingüísticas (descripción de hechos, de modelos explicativos y justificación) en las actividades dirigidas al estudio de alguno de los tópicos astronómicos. Esta conjunción de habilidades incide directamente en el conocimiento de los fenómenos astronómicos y en su interpretación, al tiempo que otorga sentido al propio conocimiento del modelo (Navarro, 2011; Shen y Confrey, 2010), porque si el modelo no se emplea para explicar los hechos, ¿̨para qué queremos que nuestros alumnos lo conozcan? Es recomendable el uso de modelos explicativos ya con niños de primaria y, por supuesto, de secundaria, aunque estos sean tentativos o todavía carezcan del rigor científico que se irá adquiriendo con el tiempo, pues permite desarrollar el pensamiento abstracto para explicar los fenómenos del mundo y acercar al alumnado la esencia de la ciencia, que no es otra que elaborar modelos que expliquen los fenómenos del mundo para actuar responsablemente en él (Harlen y Qualter, 2009; Izquierdo y Adúriz Bravo, 2003; Izquierdo y Aliberas, 2004). 
Un resultado que merece especial atención es el tratamiento comparativamente peor que recibe el estudio de los cambios astronómicos anuales. En este sentido, conviene destacar que si bien algunos profesores de primaria y de secundaria asocian distintas habilidades cognitivo-lingüísticas a este particular en las actividades de enseñanza, la justificación tiene una presencia reducida, especialmente entre el colectivo de secundaria, lo que muestra un desequilibrio en el uso de las habilidades. Además, ningún participante de los que han mostrado un nivel de adecuación alto lo ha hecho respecto al estudio de los cambios anuales. Entendemos que tal estudio es especialmente relevante y debe tratarse en secundaria, pues sobre él se han descrito abundantes preconceptos (Blown y Bryce, 2010; De Manuel y Graus, 1996; Trumper, 2001), lo que muestra su dificultad. Se reconoce que tanto la disposición de destrezas geométricas, como el dominio de una buena relación entre la perspectiva local y la espacial, puede constituir un problema incluso para el alumnado de mayor edad (Gil Quilez y Martínez Peńa, 2005; Lanciano y Camino, 2008). De ahí que se hayan promovido propuestas de enseñanza en este sentido (AA. VV., 2009; Martínez Losada y García Barros, 2013). En lo que respecta a la evaluación, los participantes exigen sobre todo la descripción de modelos y la definición en relación con los distintos tópicos, siendo los docentes de secundaria los que parecen otorgar más relevancia a la justificación asociada específicamente al estudio de la Luna y sus cambios. A pesar de que el número reducido de cuestiones de evaluación no nos permite profundizar en la adecuación de la propia evaluación, consideramos conveniente que la exigencia de la justificación se extienda y equilibre asociándola al menos a aquel tópico al que el docente otorga mayor relevancia, pues, como ya se comentó, constituye una habilidad que aglutina distintos conocimientos. Por otra parte, llama la atención que algunos docentes exijan en la evaluación ciertas habilidades que encierran un grado de dificultad alto para el nivel al que van dirigidas, como la descripción de fenómenos relativos a los cambios anuales en primaria o la propia justificación asociada a ellos o al estudio de la Luna en secundaria, sin haberlas trabajado en relación con estos aspectos en las actividades. Entendemos que esta exigencia en la evaluación debe tener un reflejo en las actividades de enseñanza, pues, aunque posiblemente el docente realice justificaciones a lo largo de explicaciones, incluso dialogadas, promoviendo la intervención del alumno, es conveniente, dada su dificultad e interés, dedicarle mayor espacio, máxime cuando otras habilidades, como por ejemplo la descripción de modelos, reciben una atención a nuestro juicio excesiva.

Finalmente, cabe destacar que las conclusiones obtenidas resultan coherentes con la valoración realizada por un amplio grupo de docentes de las habilidades cognitivo-lingüísticas asociadas al estudio de aspectos astronómicos (García Barros y Martínez Losada, 2014). Entendemos que en este trabajo hemos dado un paso más al acercarnos a las decisiones curriculares del profesorado, pues aunque los resultados haya que tomarlos con cautela, dado que el número de participantes no es muy amplio, nos ha permitido poner de manifiesto que la astronomía se trata de forma sesgada tanto en educación primaria como en secundaria. Concretamente se incide en aspectos complejos, como el estudio del sistema solar, en detrimento de otros, empleando además habilidades cognitivo-lingüísticas poco variadas, donde por ejemplo la justificación y la descripción de los hechos observables carecen de la dimensión que se merecen para propiciar el aprendizaje. En este sentido, consideramos deseable que en la formación permanente del profesorado y en la elaboración de textos escolares se subsanen estos problemas.

\section{CONSIDERACIONES FINALES}

Tomando como referente lo indicado en el apartado anterior, consideramos que sería necesario establecer una progresión más adecuada del estudio de la astronomía entre educación primaria y secundaria. En este sentido, sería conveniente emplear las habilidades cognitivo-lingüísticas de forma progresivamente más exigente aplicándolas a aspectos conceptuales cada vez más complejos y abstractos. Así, al iniciar la enseñanza primaria debería centrarse en la observación del cielo (el Sol nos da luz, produce 
sombras..., la Luna toma distintas formas, las estrellas forman constelaciones). Un segundo paso sería apreciar cambios y regularidades observables en tiempos cada vez más largos (día/mes). Paralelamente se recomendaría empezar a usar los modelos Sol-Tierra y Sol-Tierra-Luna y sus movimientos asociados para explicar los cambios observables (movimiento aparente del Sol/Luna/estrellas a lo largo del día; cambios de la Luna a lo largo del mes). Por último, un tercer nivel de dificultad recomendable para el final de primaria e inicio de la ESO sería la introducción de regularidades observables menos evidentes, pues requieren observaciones más precisas y dilatadas en el tiempo, y la justificación de estas desde un modelo teórico más complejo. En este sentido, es imprescindible responder a cuestiones del tipo: ¿cómo varía en las zonas templadas del planeta el número de horas de luz, la altura del Sol al mediodía, etc.?, para pasar a continuación a justificar sus causas empleando el modelo de traslación terrestre, dando respuesta así a cuestiones del tipo: ¿cómo se pueden explicar esos cambios?, ¿cuál es la razón de que en las zonas templadas de ambos hemisferios las estaciones no sean coincidentes?, etc. Así mismo, respecto al estudio del sistema solar, tema tan atendido por los profesores participantes, sería conveniente abordar ciertos aspectos observacionales/descriptivos que también constituyen la base de posteriores justificaciones empleando nuestro modelo planetario. Nos referimos por ejemplo a conocer y describir cómo son los planetas, qué los diferencia a simple vista de las estrellas, destacando el brillo llamativo de alguno de ellos, así como su carácter de «estrellas errantes» que no guardan posiciones fijas respecto a otras estrellas. Si bien es verdad que estos aspectos no son fácilmente observables, pueden ser descritos empleando aplicaciones informáticas de fácil acceso incluso desde el teléfono móvil. Por otra parte, el conocimiento del modelo de sistema solar no debe servir únicamente para nombrar planetas en el orden adecuado, sino para explicar los aspectos citados, incidiendo muy especialmente en la reflexión de la luz.

En definitiva, es importante que el alumnado se exprese y construya textos diferentes, con distinta intención, pues todos son necesarios. Es de sobra conocido que «saber ciencias y no saber explicarlas es como no saberlas». Por ello, las actividades deben insistir en «hablar/escribir ciencias», pues el monólogo unidireccional del docente/libro de texto se ha visto claramente insuficiente.

\section{AGRADECIMIENTOS}

Agradecemos la colaboración desinteresada e imprescindible de los profesores y profesoras participantes, sin ellos este trabajo no podría llevarse a cabo. La aportación de sus materiales encierra un encomiable valor para la mejora de la enseńanza en la que estamos directamente implicadas. Este trabajo ha sido financiado por la Xunta de Galicia (Proyecto-código INCITE08XIB106098PR).

\section{BIBLIOGRAFÍA}

AA. VV. (2009). El aprendizaje del modelo Sol-Tierra. Una oportunidad para la formación de los maestros. Alambique, 61, pp. 27-37.

BAXter, J. (1989). Children's understanding of familiar astronomical events. International Journal of Science Education, 11, pp. 505-513.

http://dx.doi.org/10.1080/0950069890110503

Bell, R.L. y Trundle, K.C. (2008). The use of a computer simulation to promote scientific conceptions of moon phases. Journal Research in Science Teaching, 45(3), pp. 346-372. http://dx.doi.org/10.1002/tea.20227

Blown, E. y Bryce, T.G.K. (2010). Conceptual Coherence Revealed in Multi-Modal Representations of Astronomy Knowledge. International Journal of Science Education, 32(1), pp. 31-67.

http://dx.doi.org/10.1080/09500690902974207 
BOE (4 de mayo de 2006). Ley Orgánica 2/2006, de 3 de mayo de Educación (LOE).

Bryce, T.G.K. y Blown, E. (2012). The Novice-Expert Continuum in Astronomy Knowledge. International Journal of Science Education, 34, pp. 545-587. http://dx.doi.org/10.1080/09500693.2011.642325

Cañas, A.; Martin-Díaz, M. y Nieda, J.(2007). Competencia en el conocimiento y la interacción con el mundo físico. La competencia cientifica. Madrid: Alianza.

Couto Candedo, P.; García Barros, S. y Martínez Losada, C. (2013). ¿Cómo son las actividades de Didáctica de las Ciencias que proponemos a los futuros maestros de primaria? Paper presented at the IX Congreso Internacional sobre Investigación en Didáctica de las Ciencias. 9-12 de septiembre de 2013. Girona.

De Manuel, J. y Graus, R. (1996). Concepciones y dificultades comunes en la construcción del pensamiento biológico. Alambique, 7, pp. 53-63.

De Pro, A. (1998). ¿'Se pueden enseñar contenidos procedimentales en als clases de ciencias? Enseñanza de las Ciencias, 16(1), pp. 21-41.

DOG (9 de julio de 2007). Decreto 130/2007, del 28 de junio, por el que se establece el DCB de Primaria.

DOG (13 de julio de 2007). DCB de la ESO: Decreto 133/2007, del 5 de julio por el que se establece el DCB de Enseñanza Secundaria Obligatoria.

García Barros, S. y Martínez Losada, C. (2013). Inmersos en el aire miramos al cielo. Los fenómenos atmosféricos y astronómicos. Barcelona: Graó.

García Barros, S. y Martínez Losada, C. (2014). La importancia de las habilidades cognitivolingüísticas asociadas al estudio de la astronomía desde la perspectiva del profesorado. Enseñanza de las Ciencias. En prensa.

García Barros, S.; Mondelo, M. y Martínez Losada, C. (1996). La Astronomía en la formación de profesores. Alambique, 10, pp. 121-127.

Gavidia Catalán, V. (2014). A vueltas con el gnomon. Buscando soluciones a problemas. Enseñanza de las Ciencias, 32(3), pp. 631-647. http://dx.doi.org/10.5565/rev/ensciencias. 1327

Gil Quilez, M.J. y Martínez Peña, B. (2005). El modelo de Sol-Tierra-Luna en el lenguaje inográfico de estudiantes de magisterio. Enseñanza de las Ciencias, 23(2), pp. 153-166.

Harlen, W. y Qualter, A. (2009). The teaching of science in primary schools: Abingdon: Routledge.

Izquierdo, M. y Adúriz Bravo, A. (2003). Epistemological Foundations of School Science. Science \& Education, 12(1), pp. 27-43. http://dx.doi.org/10.1023/A:1022698205904

Izquierdo, M. y Aliberas, J. (2004). Pensar, actuar i peular a la clase de ciències. Por un ensenyament de les ciències racional $i$ raonable.

Jiménez Alexandre, M.P. (2003). Comunicación y lenguaje en clase de ciencias. In M.P. Jiménez Alexandre (ed.). Enseñar ciencias. Barcelona: Graó, pp. 55-71.

Jiménez Alexandre, M.P. (2010). Diez ideas clave. Competencias en argumentación y uso de pruebas. Barcelona: Graó.

Jorba, J. (2000). La comunicación y las habilidades cognitivo-lingüísticas. In J. Jorba, I. Gómez y A. Prats (eds.). Hablar y escribir para aprender. Uso de la lengua en situación de enseñanza-aprendizaje desde las áreas curriculares. Barcelona: ICE Universitat Autònoma de Barcelona. Síntesis, pp. 29-49.

Lanciano, N. y Camino, N. (2008). Del ángulo de la geometría a los ángulos en el cielo. Dificultades para la conceptualización de las coordenadas astronómicas acimut y altura. Enseñanza de las Ciencias, 26(1), pp. 77-92. 
Lelliott, A. y Rollnick, M. (2010). Big Ideas: A review of astronomy education research 19742008. International Journal of Science Education, 32, pp. 1771-1799.

http://dx.doi.org/10.1080/09500690903214546

Martínez Losada, C. y García Barros, S. (2013). Habilidades cognitivo-lingüísticas y nutrición humana en la formación inicial del profesorado de secundaria. Revista Eureka sobre Enseñanza y Divulgación de las Ciencias, 10 (extraordinario), pp. 664-677.

Mellado, V. (2001). ¿Por qué a los profesores de Ciencias nos cuesta tanto cambiar nuestras concepciones y modelos didácticos? Revista Interuniversitaria de Formación del Profesorado, 40, pp. 17-30.

Navarrete, A. (2003). Obstáculos y dificultades en la evolución de las estructuras conceptuales y epistemológicas de los futuros maestros: Un estudio de caso sobre el fenómeno de las estaciones. Cádiz: Universidad de Cádiz.

NaVArro, M. (2011). Enseñanza y aprendizaje de astronomía diurna en primaria mediante «secuencias problematizadas» basadas en «mapas evolutivos». Enseñanza de las Ciencias, 29(2), pp. 163-174.

Nussbaum, J. (1979). Children's conception of the Earth as a cosmic body: a cross-age study. Science Education, 63(1), pp. 83-93. http://dx.doi.org/10.1002/sce.3730630113

Ospina Quintero, N. y Bonan, L. (2011). Explicaciones y argumentos de profesores de quimica en formación inicial: la construcción de criterios para su evaluación. Eureka sobre enseñanza y divulgación de las ciencias, 8, pp. 2-19.

Palomar Fons, R. (2014). Enseñanza y aprendizaje de la Astronomía en el bachillerato. Tesis doctoral. Enseñanza de las Ciencias, 32(3), pp. 699-700. http://dx.doi.org/10.5565/rev/ensciencias. 1460

Pérez Esteve, P. y Zayas, F. (2007). Competencia en comunicación lingüística. Madrid: Alianza Editorial.

Plummer, J.D. (2009). A cross-age study of children's knowledge of apparent celestial motion. International Journal of Science Education, 31, pp. 1571-1606. http://dx.doi.org/10.1080/09500690802126635

Plummer, J.D.; Waskoa, K.D. y Slagleb C. (2011). Children Learning to Explain Daily Celestial Motion: Understanding astronomy across moving frames of reference. International Journal of Science Education, 33, pp. 1963-1992. http://dx.doi.org/10.1080/09500693.2010.537707

Porlan, R.; Martin del Pozo, R.; Rivero, A.; Harres, J.; Azcárate, P. y Pizzato, M. (2010). El cambio del profesorado de ciencias I: Marco Teórico y Formativo. Enseñanza de las Ciencias, 28(1), pp. 31-46.

PraIn, V. (2006). Learning from writing in secondary science: Some theoretical and practical implications. International Journal of Science Education, 28(2-3), pp. 179-201. http://dx.doi.org/10.1080/09500690500336643

SANmartí, N. (2007). Hablar, leer y escribir para aprender ciencia. In P. Fernández (ed.). La competencia en comunicación lingüistica en las áreas del curriculo. Madrid: Colección Aulas de Verano. MEC.

Shen, J. y Confrey, J. (2007). From conceptual change to transformative modeling: A case study of an elementary teacher in learning astronomy. Science Education, 91(6), pp. 948-966. http://dx.doi.org/10.1002/sce.20224

Shen, J. y Confrey, J. (2010). Justifying alternative models in learning astronomy: A study of k-8 science teachers' understanding of frames of reference. International Journal of Science Education, 32(1), pp. 1-29.

Sutton, C. (2003). Los profesores de ciencias como profesores de lenguaje. Enseñanza de las Ciencias, 21(1), pp. 21-25. 
Trumper, R. (2001). A cross-age study of junior high school students' conceptions of basic a concepts. International Journal of Science Education, 23(11), pp. 1111-1123.

http://dx.doi.org/10.1080/09500690010025085

Trumper, R. (2006). Teaching Future Teachers Basic Astronomy Concepts-Seasonal Changes-at a time of Reform in Science Education. Journal of Research in Science Teaching, 43(9), pp. 879-906.

VÁzquez, A.; Pelizza, L.; Jacobi y Rosales, P. (2008). Concepciones de los profesores universitarios acerca de las relaciones entre tareas de escritura y aprendizaje. Disponible en línea: <http://www. filo.unt.edu.ar/jorn_unesco/cd/PAN\%202.3.VAZQUEZ.pdf>.

Vega Navarro, A. (2001). Tenerife tiene seguro de sol (y de luna): representaciones del profesorado de primaria acerca del día y la noche. Enseñanza de las Ciencias, 19(1), pp. 31-44.

Vosniadou, S. y Brewer, W.F. (1992). Mental models of the earth:a study of conceptual change in childgood. Cognitive Psicology, 24, pp. 535-585.

http://dx.doi.org/10.1016/0010-0285(92)90018-W 


\title{
What content and which cognitive-linguistic skills are used by primary and secondary school teachers when teaching astronomy
}

\author{
Concepción González Rodríguez, Susana García Barros, Cristina Martínez Losada \\ Facultade de Ciencias da Educación. Universidade da Coruña. España. \\ cgonzalezr@udc.es, susg@udc.es y cmarl@udc.es
}

The aim of this study is to investigate further into the thinking of a group of teachers working at a Primary and Secondary Education level, in order to find out more about their curricular decisions. Specifically, the objective is to respond to the following questions: which aspects of astronomy does a small group of primary and secondary school teachers work on in their teaching activities and which of these aspects do they assess? Which cognitive-linguistic skills do they require in such aspects? Is any sort of relationship established between the use of cognitive-linguistic skills and the specific aspects of astronomy? Is the work on these skills balanced compared to such aspects?

To do this, an analysis was carried out on a total of 373 teaching activities and 103 assessment questions, both of which were aimed at the study of astronomy in Compulsory Education and provided by 14 teachers (7 from primary schools and 7 from secondary schools). The activities and the assessment questions were used by each teacher in astronomy classes during the academic year in which this study took place.

The analysis of such materials was focused on the theoretical aspects of astronomy and the cognitive-linguistic skills they require. Specifically, four categories were established for the study of aspects of astronomy: $a$ ) daily astronomical changes; $b$ ) monthly astronomical changes - the study of the Moon; $c$ ) annual astronomical changes; and $d$ ) the Earth in space - planets of the solar system, galaxies, etc. On the other hand, in the study of skills, the focus was on the description of observable phenomena, the description of theoretical models and the definition and justification of events/phenomena.

The results from the first research question showed that the participants covered the various categories but tended to insist on the more complex aspect - the Earth in space, both in the activities and in the assessment questions. It was also observed that the primary school teachers dedicated proportionately fewer activities to the study of the closest and most appreciable astronomical aspects, showing that there is no clear progress from Primary to Secondary.

With regard to the second question, referring to the cognitive-linguistic skills required by the activities and assessment questions, it was detected that there was greater variety of skills in the latter. Also noted was a preponderance of descriptions of theoretical models (Sun/Earth, Sun/Earth/Moon, the solar system), with the description of observable phenomena being less frequent. Justification was a skill used more widely by secondary school teachers, perhaps due to the fact that secondary education is more demanding and requires knowledge and use of theoretical models.

With regard to the last two research questions, it was noticed that the behaviour of the participants was unequal. Primary school teachers seemed to apply cognitive-linguistic skills more intensely to specific topics. Therefore, the description of observable phenomena was above all associated with the study of the lunar cycle, the definition of the planetary system and the justification of daily astronomical changes. In contrast, secondary school teachers tended to use a greater variety of cognitive-linguistic skills, associating them more or less equally to the various astronomical topics.

In general terms, this study has allowed us to highlight the fact that the teaching of astronomy still stresses the complex aspects, such as the study of the solar system (model of the planetary system), as opposed to other aspects which are closer and simpler (astronomy which is observable in the sky). Furthermore, the cognitive-linguistic skills that are used are not varied and therefore the description of observable events and their corresponding justifications, using theoretical models, should be given more importance in the activities, so as to promote the appropriate learning. As a result, we believe it would be desirable for these problems to be resolved during permanent teacher training and in the creation of text books. 
